\title{
Revisiting the axial anomaly for light mesons and baryons
}

\author{
Francesco Giacosa ${ }^{* \dagger}$ \\ Institute of Physics, Jan Kochanowski University, ul. Swietokrzyska 15, 25-406 Kielce \\ Institute for Theoretical Physics, Johann Wolfgang Goethe University, Max-von-Laue-Str. 1, \\ 60438 Frankfurt am Main \\ E-mail: fgiacosa@ujk.edu.pl
}

The axial anomaly is responsible for the masses and mixing of the mesons $\eta$ and $\eta^{\prime}$. An open question is if (and to what extent) it affects also other hadrons. We show that anomalous terms can be important to understand the spectroscopy of the pseudotensor mesons $\eta_{2}(1645)$ and $\eta_{2}(1870)$. In fact, pseudotensor mesons belong to a so-called heterochiral multiplet, for which a quadratic mixing term between nonstrange and strange isoscalar members arises. On the contrary, for so-called homochiral multiplets, such as the ground-state (axial-)vector and tensor mesons, this mixing is not possible, hence one can easily understand why the isoscalar members of these multiplets are almost purely nonstrange and strange, respectively. Moreover, the axial anomaly can be also coupled to baryons (within the mirror assignment), and thus it helps to explain the large decay width $N^{*}(1535) \rightarrow N \eta$ and to clarify which baryons are chiral partners.

XVII International Conference on Hadron Spectroscopy and Structure - Hadron2017 25-29 September, 2017

University of Salamanca, Salamanca, Spain

\footnotetext{
* Speaker.

${ }^{\dagger}$ A footnote may follow.
} 


\section{Introduction}

In the chiral limit, the QCD Lagrangian contains a $U(1)_{A}$ symmetry, which is broken by quantum fluctuations: this is the so-called axial anomaly [1]. Its role is necessary to understand the properties of the mesons $\eta \equiv \eta(547)$ and $\eta^{\prime} \equiv \eta^{\prime}$ (958) (especially the mass of $\eta^{\prime}$ and the large mixing between nonstrange and strange contributions) [2]. Yet, what about other mesons? And what about the role of the axial anomaly for baryons? In these proceedings, we report on two recent works in which the role of the axial anomaly was studied in the mesonic and baryonic sectors $[3,4]$.

In sec. 2, based on Ref. [3], light meson nonets are grouped into chiral multiplets and classified according to their chiral transformations: there are "heterochiral" mesons (such as the (pseudo)scalar states) and 'homochiral' mesons (such as (axial-)vector states). For all heterochiral multiplets, the axial anomaly allows for mixing of nonstrange and strange isoscalar members, as well known for the $\eta$ and $\eta^{\prime}$ mesons mentioned above. In addition, such a mixing seems to be realized also for heterochiral pseudotensor mesons [5]. On the contrary, this anomalous mixing is not possible for "homochiral" multiplets: one can easily understand why the vector mesons $\omega(782)$ and $\phi(1020)$ as well as the tensor mesons $f_{2}(1270)$ and $f_{2}^{\prime}(1525)$ are (almost purely) nonstrange and strange, respectively.

In Sec. 3, based on Ref. [4], the axial anomaly is used to understand the anomalously large decay $N(1535) \rightarrow N \eta$ (much larger than than what flavor symmetry naively predicts) as well as the decay $\Lambda(1670) \rightarrow \Lambda(1116) \eta$. Namely, the axial anomaly allows for a chiral anomalous term which couples baryonic chiral partners to the mesons $\eta$ and $\eta^{\prime}$. As a consequence, this study also shows that $N(1535)$ is predominantly the chiral partner of $N(939)$, and $\Lambda(1670)$ the chiral partner of $\Lambda(1116)$.

\section{Axial anomaly in the light mesonic sector}

Let us start with the (pseudo)scalar sector. For three flavors $\left(N_{f}=3\right)$ the corresponding mesonic matrix $\Phi$ (with elements $\Phi^{i j}=\bar{q}_{\mathrm{R}}^{j} q_{\mathrm{L}}^{i}$ ) reads

$$
\Phi=S+i P=\frac{1}{\sqrt{2}}\left(\begin{array}{ccc}
\frac{\sigma_{N}+a_{0}^{0}}{\sqrt{2}} & a_{0}^{+} & K_{0}^{*+} \\
a_{0}^{-} & \frac{\sigma_{N}-a_{0}^{0}}{\sqrt{2}} & K_{0}^{* 0} \\
K_{0}^{*-} & K_{0}^{* 0} & \sigma_{S}
\end{array}\right)+\frac{1}{\sqrt{2}}\left(\begin{array}{ccc}
\frac{\eta_{N}+\pi^{0}}{\sqrt{2}} & \pi^{+} & K^{+} \\
\pi^{-} & \frac{\eta_{N}-\pi^{0}}{\sqrt{2}} & K^{0} \\
K^{-} & \bar{K}^{0} & \eta_{S}
\end{array}\right),
$$

where $a_{0} \equiv a_{0}(1450)$ and $K_{0}^{*} \equiv K_{0}^{*}(1430)$. In addition, $\sigma_{N}$ corresponds predominantly to $f_{0}(1370)$ and $\sigma_{S}$ to $f_{0}(1500)$ (with admixture among each other and with a bare scalar glueball in $f_{0}(1710)$ [6]). In the pseudoscalar sector, $\vec{\pi}$ are the pions and $K^{ \pm}, K^{0}, \bar{K}^{0}$ the kaons, while $\eta_{N} \equiv \sqrt{1 / 2}(\bar{u} u+$ $\bar{d} d)$ and $\eta_{S} \equiv \bar{s} s$ are the nonstrange and strange counterparts of $\eta \equiv \eta(547)$ and $\eta^{\prime} \equiv \eta^{\prime}(958)$, see below.

By applying the chiral transformation $S U(3)_{R} \times S U(3)_{L} \times U(1)_{A}\left(\right.$ which reads $q_{\mathrm{L} / \mathrm{R}} \rightarrow\left(\mathrm{e}^{\mp \mathrm{i} \alpha / 2} U_{\mathrm{L} / \mathrm{R}}\right) q_{\mathrm{L} / \mathrm{R}}$, $\alpha$ refers to $\left.U(1)_{A}\right)$, the matrix $\Phi$ transforms as

$$
\Phi \rightarrow e^{-i \alpha} U_{L} \Phi U_{R}^{\dagger}
$$


hence the name heterochiral: it picks up both matrices $U_{L}$ and $U_{R}$. Generally, terms of a chiral model (e.g. [6, 7]) are powers of $\Phi^{\dagger} \Phi$, such as $\operatorname{tr}\left(\Phi^{\dagger} \Phi\right), \operatorname{tr}\left(\Phi^{\dagger} \Phi\right)^{2}$, ..., thus invariant under the chiral transformation $S U(3)_{R} \times S U(3)_{L}$ and also under the axial transformation $U(1)_{\mathrm{A}}$. However, there are other possibilities: one can use the property of the determinant that under $S U(3)_{R} \times$ $S U(3)_{L} \times U(1)_{A}$ transforms as

$$
\operatorname{det}(\Phi)=\frac{1}{6} \varepsilon^{i j k} \varepsilon^{i^{\prime} j^{\prime} k^{\prime}} \Phi^{i i^{\prime}} \Phi^{j j^{\prime}} \Phi^{k k^{\prime}} \rightarrow \mathrm{e}^{-3 \mathrm{i} \alpha} \operatorname{det}(\Phi)
$$

in order to build anomalous terms invariant under $S U(3)_{R} \times S U(3)_{L}$ but not under $U(1)_{A}$, such as:

$$
\mathscr{L}_{\Phi}^{\text {anomaly }}=-a_{\mathrm{A}}^{(3)}\left[\operatorname{det}(\Phi)-\operatorname{det}\left(\Phi^{\dagger}\right)\right]^{2}+\ldots
$$

(dots refer to other terms [3]). Here, for simplicity we keep only the "third" term of that work (by using the same notation). This term gives a contribution to the effective potential of the form $-\alpha_{\mathrm{A}} \eta_{0}^{2}=-\alpha_{\mathrm{A}}\left(\sqrt{2} \eta_{N}+\eta_{S}\right)^{2}$ with $\alpha_{\mathrm{A}} \simeq a_{\mathrm{A}}^{(3)} \phi_{N}^{2}$. This term, which was also obtained in Ref. [7] and naturally arises when integrating out a pseudoscalar glueball [8], clearly affects the mixing in the pseudoscalar sector (but not in the scalar sector). The physical fields $\eta \equiv \eta(547)$ and $\eta^{\prime} \equiv$ $\eta^{\prime}(958)$ are

$$
\eta=\eta_{N} \cos \theta_{P}+\eta_{S} \sin \theta_{P}, \eta^{\prime}=-\eta_{N} \sin \theta_{P}+\eta_{S} \cos \theta_{P} .
$$

The pseudoscalar mixing angle $\theta_{P}$ reads $[2,3]$ :

$$
\theta_{P}=-\frac{1}{2} \arctan \left[\frac{4 \sqrt{2} \alpha_{\mathrm{A}}}{2\left(m_{K}^{2}-m_{\pi}^{2}-\alpha_{\mathrm{A}}\right)}\right] ;
$$

$\theta_{P}$ is negative for realistic values of $\alpha_{\mathrm{A}}$. The term of Eq. (2.4) alone is not sufficient for a precise description of the axial anomaly, but allows to understand its most salient phenomenological features. Numerically, $\theta_{P}$ varies between $-40^{\circ}$ and $-45^{\circ}$ [2].

Next, we move to (axial-)vector mesons, described by the matrices $V_{\mu}$ (with $J^{P C}=1^{--}$, corresponding to the resonances $\left\{\rho(770), K^{*}(892), \omega(782), \phi(1020)\right\}$ ) and $A_{\mu}$ (with $J^{P C}=1^{++}$, $\left.\left\{a_{1}(1260), K_{1, A}, f_{1}(1285), f_{1}(1420)\right\}\right)$, see also the PDG for details [9]. The chiral objects are the right-handed and the left-handed currents $R_{\mu}^{i j}=\bar{q}_{\mathrm{R}}^{j} \gamma_{\mu} q_{\mathrm{R}}^{i}, L_{\mu}^{i j} \equiv \bar{q}_{\mathrm{L}}^{j} \gamma_{\mu} q_{\mathrm{L}}^{i}$ with $R_{\mu}=V_{\mu}-A_{\mu}$ and $L_{\mu}=V_{\mu}+A_{\mu}$. Under $S U(3)_{R} \times S U(3)_{L} \times U(1)_{A}$ :

$$
L_{\mu} \longrightarrow U_{\mathrm{L}} R_{\mu} U_{\mathrm{L}}^{\dagger}, R_{\mu} \longrightarrow U_{\mathrm{R}} R_{\mu} U_{\mathrm{R}}^{\dagger}
$$

therefore these multiplets are named homochiral (either only $U_{\mathrm{L}}$ or only $U_{\mathrm{R}}$ enter, respectively). Here it is not possible to write down a term such as in Eq. (2.4). (Other more complicated WessZumino terms, see e.g. Ref. [10], exist but to not affect the isoscalar mixing). As a consequence, we expect the isoscalar mixing to be much suppressed. This is in very good agreement with observations: the resonance $\omega(782)$ is almost purely nonstrange, while $\phi(1020)$ almost purely strange. Similarly, $f(1285)$ is predominantly nonstrange and $f_{1}(1420)$ strange.

Ground-state tensor mesons $\left(J^{P C}=2^{++},\left\{a_{2}(1320), K_{2}^{*}(1430), f_{2}(1270), f_{2}^{\prime}(1525)\right\}\right)$, represent also a very well-known nonet of $\bar{q} q$ states [11]. Together with their not yet known chiral partners $\left(J^{P C}=2^{--}\right.$, only $K_{2}(1820)$ has been discovered), they are described by the heterochiral multiplet $L_{\mu v}^{i j}=\bar{q}_{\mathrm{L}}^{j}\left(\gamma_{\mu} \overleftrightarrow{D_{v}}+\gamma_{v} \overleftrightarrow{D_{\mu}}+\ldots\right) q_{\mathrm{L}}^{i}, R_{\mu \nu}^{i j} \equiv \bar{q}_{\mathrm{R}}^{j}\left(\gamma_{\mu} \overleftrightarrow{D_{v}}+\gamma_{v} \overleftrightarrow{D_{\mu}}+\ldots\right) q_{\mathrm{R}}^{i}$ with $D_{\mu}$ being the covariant 
derivative. (The tensor states are $\left(L_{\mu \nu}+R_{\mu \nu}\right) / 2$ ). The currents transform as $L_{\mu \nu} \longrightarrow U_{\mathrm{L}} L_{\mu \nu} U_{\mathrm{L}}^{\dagger}$ and $R_{\mu \nu} \longrightarrow U_{\mathrm{L}} R_{\mu \nu} U_{\mathrm{L}}^{\dagger}$, hence we are again in presence of a homochiral multiplet. Just as before, we expect a small isoscalar mixing, a fact which is very well confirmed by experiments: $f_{2}(1270)$ is to a very good extent purely nonstrange and $f_{2}^{\prime}(1525)$ strange.

We now move to heterochiral vectors, which contain the pseudovector states $P_{\mu} \equiv\left\{b_{1}(1235)\right.$, $\left.K_{1, B}, h_{1}(1170), h_{1}(1380)\right\}$ with $J^{P C}=1^{+-}$and their chiral partners, the orbitally excited vector mesons $S_{\mu} \equiv\left\{\rho(1700), K^{*}(1680), \omega(1650), \phi(? ? ?)\right\}$ with $J^{P C}=1^{--}$(see also Ref. [12] where the identification $\phi(? ? ?) \equiv \phi(1930)$ was put forward). To this end, we construct the object $\Phi_{\mu}^{i j} \equiv$ $\bar{q}_{\mathrm{R}}^{j}\left(\overleftrightarrow{D_{\mu}}+\ldots\right) q_{\mathrm{L}}^{i}=S_{\mu}+\mathrm{i} P_{\mu}$, which transforms as $\Phi_{\mu} \rightarrow \mathrm{e}^{-\mathrm{i} \alpha} U_{\mathrm{L}} \Phi_{\mu} U_{\mathrm{R}}^{\dagger}$, i.e. just as (pseudo)scalar mesons. The anomalous Lagrangian (keeping only the term analogous to Eq. (2.4)) reads

$$
\mathscr{L}_{\Phi_{\mu}}^{\text {anomaly }}=-b_{\mathrm{A}}^{(3)}\left(\varepsilon^{i j k} \varepsilon^{i^{\prime} j^{\prime} k^{\prime}} \Phi^{i i^{\prime}} \Phi^{j j^{\prime}} \Phi_{\mu}^{k k^{\prime}}-\text { h.c. }\right)^{2}+\ldots
$$

which reduces to $-\beta_{\mathrm{A}}\left(\sqrt{2} h_{1, N}^{\mu}+h_{1, S}^{\mu}\right)^{2}$, with $\beta_{\mathrm{A}} \simeq b_{\mathrm{A}}^{(3)} \phi_{N}^{2}$, when quadratic terms are considered. Here, a large mixing between the nonstrange $h_{1, N}^{\mu}$ and the strange $h_{1, S}^{\mu}$ components is possible. Unfortunately, the present experimental knowledge of the physical states is very poor $\left(h_{1}(1380)\right.$ is still omitted from the summary of the PDG), thus a verification of their mixing is not possible. It will be interesting in the future to study these states in more detail.

Last, and most interestingly, we study the heterochiral tensors. They include the nonet of pseudotensor mesons $P_{\mu v} \equiv\left\{\pi_{2}(1670), K_{2}(1770), \eta_{2}(1645), \eta_{2}(1870)\right\}$ with $J^{P C}=2^{-+}$(see also Ref. [14]) as well as their not yet known chiral partners $S_{\mu v}$ with $J^{P C}=2^{++}$. The chiral object is $\Phi_{\mu v}=S_{\mu v}+\mathrm{i} P_{\mu \nu} \equiv \bar{q}_{\mathrm{R}}^{j}\left(\overleftrightarrow{D_{\mu}} \overleftrightarrow{D_{v}}+\ldots\right) q_{\mathrm{L}}^{i}$, which transforms as $\Phi_{\mu v} \rightarrow \mathrm{e}^{-\mathrm{i} \alpha} U_{\mathrm{L}} \Phi_{\mu \nu} U_{\mathrm{R}}^{\dagger}$, thus just as the (pseudo)scalars. The anomalous Lagrangian (keeping only the term analogous to Eq. (2.4)) reads

$$
\mathscr{L}_{\Phi_{\mu \nu}}^{\text {anomaly }}=c_{\mathrm{A}}^{(3)}\left(\varepsilon^{i j k} \varepsilon^{i^{\prime} j^{\prime} k^{\prime}} \Phi^{i i^{\prime}} \Phi^{j j^{\prime}} \Phi_{\mu \nu}^{k k^{\prime}}-\text { h.c. }\right)^{2}+\ldots,
$$

that reduces to $-\gamma_{A}\left(\sqrt{2} \eta_{2, N}^{\mu v}+\eta_{2, S}^{\mu v}\right)^{2}$ with $\gamma_{A} \simeq c_{\mathrm{A}}^{(3)} \phi_{N}^{2}$ for quadratic terms. Interestingly, the phenomenological study of Ref. [5] found that the physical states are

$$
\left(\begin{array}{l}
\eta_{2}(1645) \\
\eta_{2}(1870)
\end{array}\right)=\left(\begin{array}{cc}
\cos \theta_{P T} & \sin \theta_{P T} \\
-\sin \theta_{P T} & \cos \theta_{P T}
\end{array}\right)\left(\begin{array}{c}
\eta_{2, N}=\frac{\bar{u} u+\bar{d} d}{\sqrt{2}} \\
\eta_{2, S}=\bar{s} s
\end{array}\right) \text { with } \theta_{P T} \simeq-42^{\circ},
$$

which is a surprisingly large mixing. This result can be nicely explained by the axial anomaly being active in this sector. Moreover, the corresponding mixing angle turns out to be negative (for realistic values of $\gamma_{\mathrm{A}}$ ) just as in the (pseudo)scalar sector:

$$
\theta_{P T} \simeq-\frac{1}{2} \arctan \left[\frac{4 \sqrt{2} \gamma_{\mathrm{A}}}{2\left(m_{K_{2}(1770)}^{2}-m_{\pi_{2}(1660)}^{2}-\gamma_{\mathrm{A}}\right)}\right]<0 .
$$

Again, such an expression is only approximate since other anomalous terms are neglected, but the main point is that, just as for $\eta$ and $\eta^{\prime}$, the anomaly allows for a large mixing. Future experimental work at the ongoing Jefferson lab can investigate these resonances [13]. 


\section{Axial anomaly in the light baryonic sector}

The object ( $\left.\operatorname{det} \Phi-\operatorname{det} \Phi^{\dagger}\right)$, properly coupled to baryons, affects some decay channels, most notably $N(1535) \rightarrow N \eta$. To this end, the mirror assignment for the baryons multiplets is needed [15]. Here, we shall use the $N_{f}=3$ version of the mirror assignment presented in Ref. [16]. The physical fields are

$$
\begin{aligned}
B_{N} & \equiv\{N(939), \Lambda(1116), \Sigma(1193), \Xi(1318)\}, B_{M} \equiv\{N(1440), \Lambda(1600), \Sigma(1660), \Xi(1690)\}, \\
B_{M *} & \equiv\{N(1535), \Lambda(1670), \Sigma(1620), \Xi(?)\}, B_{N *} \equiv\{N(1650), \Lambda(1800), \Sigma(1750), \Xi(?)\},
\end{aligned}
$$

with the splitting into chiral baryonic multiplets

$$
B_{N}=\frac{N_{1}-N_{2}}{\sqrt{2}}, B_{N *}=\frac{N_{1}+N_{2}}{\sqrt{2}}, B_{M}=\frac{M_{1}-M_{2}}{\sqrt{2}}, B_{M *}=\frac{M_{1}+M_{2}}{\sqrt{2}},
$$

which transform under chiral transformations $S U(3)_{R} \times S U(3)_{L}$ as

$$
N_{1 R(L)} \rightarrow U_{R(L)} N_{1 R(L)} U_{R}^{\dagger}, N_{2 R(L)} \rightarrow U_{R(L)} N_{2 R(L)} U_{L}^{\dagger}, M_{1 R(L)} \rightarrow U_{L(R)} M_{1 R(L)} U_{R}^{\dagger}, M_{2 R(L)} \rightarrow U_{L(R)} M_{2 R(L)} U_{L}^{\dagger}
$$

The chirally symmetric but axial anomalous Lagrangian reads [4]:

$$
\begin{aligned}
\mathscr{L}_{A}^{N_{f}=3} & =\lambda_{A 1}\left(\operatorname{det} \Phi-\operatorname{det} \Phi^{\dagger}\right) \operatorname{Tr}\left(\bar{M}_{1 R} N_{1 L}-\bar{N}_{1 L} M_{1 R}-\bar{M}_{2 L} N_{2 R}+\bar{N}_{2 R} M_{2 L}\right) \\
& +\lambda_{A 2}\left(\operatorname{det} \Phi-\operatorname{det} \Phi^{\dagger}\right) \operatorname{Tr}\left(\bar{M}_{1 L} N_{1 R}-\bar{N}_{1 R} M_{1 L}-\bar{M}_{2 R} N_{2 L}+\bar{N}_{2 L} M_{2 R}\right) .
\end{aligned}
$$

Considering that $\operatorname{det} \Phi-\operatorname{det} \Phi^{\dagger} \propto i\left(\sqrt{2} \eta_{N}+\eta_{S}\right)+\ldots$, the anomaly gives contributions in which $B_{N}$ and $B_{M_{*}}$ (as well as $B_{M}$ and $B_{N_{*}}$ ) couple to $\eta_{0}$, therefore an enhanced decay of the type $B_{M_{*}} \rightarrow B_{N} \eta$ follows. In particular, the anomalously large decay $N(1535) \rightarrow N \eta$ show that $N(1535)$ and $N(939)$ are chiral partners. In addition, also the decay $\Lambda(1670) \rightarrow \Lambda \eta$ and the coupling of $N(1535)$ to $N \eta^{\prime}$ can be correctly described, see Ref. [4] for further details.

In the end, we recall that the axial anomaly is also important to describe the decays of the not-yet discovered pseudoscalar glueball. In fact, this state couples through the axial anomaly to both mesons and baryons $[4,8]$.

\section{Conclusions}

In this work we have revisited the role of the axial anomaly for what concerns the phenomenology of the light mesonic and baryonic sectors. In the mesonic sector one can -for the so-called "heterochiral" multiplets [3]- write down anomalous terms similar to the one which are usually introduced for (pseudo)scalar mesons. Hence, a large strange-nonstrange mixing in the pseudotensor nonet and in the pseudovector nonet are possible (in the former some experimental evidence already exists [5]). On the contrary, the ground-state vector and tensor mesons are "homochiral", therefore the isoscalar states are (almost purely) nonstrange and strange, respectively.

In the baryonic sector, the axial anomaly can help to understand some decay processes involving the $\eta$ meson, most notably $N(1535) \rightarrow N \eta$ [4] In fact, an anomalous term which connects baryonic chiral partners to $\eta$ and $\eta^{\prime}$ is possible when the mirror assignment is used. In turn, this approach shows that $N$ and $N(1535)$ are predominantly chiral partners. 
Acknowledgments: the author thanks R. Pisarski, A. Koenigstein, L. Olbrich, M. Zetenyi, and D. H. Rischke for cooperation. Financial support from the Polish National Science Centre NCN through the OPUS project no. 2015/17/B/ST2/01625 and from the DFG under grant no. RI 1181/6-1 are aknnowledged.

\section{References}

[1] G. 't Hooft, Phys. Rept. 142 (1986) 357. D. J. Gross, S. B. Treiman and F. Wilczek, Phys. Rev. D 19 (1979) 2188. S. L. Adler, Phys. Rev. 177 (1969) 2426. J. S. Bell and R. Jackiw, Nuovo Cim. A 60 (1969) 47.

[2] T. Feldmann, P. Kroll and B. Stech, Phys. Rev. D 58 (1998) 114006. S. D. Bass and A. W. Thomas, Phys. Lett. B 634 (2006) 368. G. Amelino-Camelia et al., Eur. Phys. J. C 68 (2010) 619. B. Borasoy and R. Nissler, Eur. Phys. J. A 26 (2005) 383.

[3] F. Giacosa, A. Koenigstein and R. D. Pisarski, arXiv:1709.07454 [hep-ph].

[4] L. Olbrich, M. Zétényi, F. Giacosa and D. H. Rischke, arXiv:1708.01061 [hep-ph].

[5] A. Koenigstein and F. Giacosa, Eur. Phys. J. A 52 (2016) no.12.

[6] D. Parganlija, P. Kovacs, G. Wolf, F. Giacosa and D. H. Rischke, Phys. Rev. D 87 (2013) no.1, 014011. S. Janowski, F. Giacosa and D. H. Rischke, Phys. Rev. D 90 (2014) no.11, 114005.

[7] C. Rosenzweig, J. Schechter and C. G. Trahern, Phys. Rev. D 21 (1980) 3388. A. H. Fariborz, R. Jora and J. Schechter, Phys. Rev. D 72 (2005).

[8] W. I. Eshraim, S. Janowski, F. Giacosa and D. H. Rischke, Phys. Rev. D 87 (2013) no.5, 054036. W. I. Eshraim, S. Janowski, A. Peters, K. Neuschwander and F. Giacosa, Acta Phys. Polon. Supp. 5, 1101 (2012).

[9] C. Patrignani et al. [Particle Data Group], Chin. Phys. C 40 (2016) no.10, 100001.

[10] H. Gomm, O. Kaymakcalan and J. Schechter, Phys. Rev. D 30 (1984) 2345.

[11] L. Burakovsky and J. T. Goldman, Phys. Rev. D 57 (1998) 2879. V. Cirigliano, G. Ecker, H. Neufeld and A. Pich, JHEP 0306 (2003) 012. F. Giacosa, T. Gutsche, V. E. Lyubovitskij and A. Faessler, Phys. Rev. D 72 (2005) 114021.

[12] M. Piotrowska, C. Reisinger and F. Giacosa, Phys. Rev. D 96 (2017) no.5, 054033.

[13] H. Al Ghoul et al. [GlueX Collaboration], AIP Conf. Proc. 1735 (2016) 020001. A. Rizzo [CLAS Collaboration], PoS CD 15 (2016) 060.

[14] B. Wang, C. Q. Pang, X. Liu and T. Matsuki, Phys. Rev. D 91 (2015) no.1, 014025. A. V. Anisovich, C. J. Batty, D. V. Bugg, V. A. Nikonov and A. V. Sarantsev, Eur. Phys. J. C 71 (2011) 1511.

[15] C. E. DeTar and T. Kunihiro, Phys. Rev. D 39, 2805 (1989). D. Jido, M. Oka, and A. Hosaka, Prog. Theor. Phys. 106, 873 (2001). C. Sasaki and I. Mishustin, Phys. Rev. C 82, 035204 (2010).

[16] L. Olbrich, M. Zetenyi, F. Giacosa and D. H. Rischke, Phys. Rev. D 93 no. 3, 034021 (2016). 